\title{
New Physics-Based Turbocharger Data-Maps Extrapolation Algorithms: Validation on a Spark-Ignited Engine
}

\author{
J. El Hadef ***, G. Colin*, V.Talon**, Y.Chamaillard* \\ *Laboratoire PRISME, 8 rue Léonard de Vinci, 45072 Orléans cédex 2, FRANCE (Tel: +33238494383; e-mail: jamil.el- \\ hadef@etu.univ-orleans.fr). \\ ** Renault SA - CTL, 1 Allée de Cornuel, 91510 Lardy, FRANCE (Tel: +33611527597; e-mail: vincent.talon@renault.com)
}

\begin{abstract}
Objectives in terms of pollutant emissions and fuel consumption reduction, as well as development costs and time to market reduction, has led car manufacturers to use more and more system simulation. However, among all the fields in which it has enabled to achieve these goals, the control development stage is one of those, in which major improvements can still be achieved. In this context and with the increasing penetration of downsized engines, turbocharger modeling has become one of the biggest challenges in engine simulation. This paper focuses on the validation of compressor and turbine data maps, extrapolated using new physics-based extrapolation algorithms. The study led to excellent prediction performances for two classical control-oriented models. Conclusions stresses: 1- The improvement of the extrapolation robustness, in particular in the low turbocharger rotational speeds zone. 2- The possibility to keep a low calculation time as well as maintaining the same calibration effort.
\end{abstract}

Keywords: Turbocharger, data-maps, interpolation, extrapolation, validation, transients, steady-state.

\section{INTRODUCTION}

Always more drastic pollutant emission standards constrained the car manufacturers to reduce the fuel consumption and pollutant emissions of internal combustion engines. This can be achieved by reducing the engine displacement as well as adding a turbocharger to the air path in order to maintain the same driving performances. In this context, model-based development strategies are a very promising way to deal with this increasing complication of engines technical definition (Gissinger et al., (2002), Dauron, (2007), Guzzella et al., (2004)). In fact, model-based development strategies such as validation on virtual test bench as well as model-embedded control are now widely integrated in car manufacturers' development processes and research programs.

In the case of turbocharged engines, the turbocharger submodel accuracy represents the biggest challenge. Usually, for calculation time considerations, it relies on extrapolated manufacturer's data maps. The goal of this study is to confirm that new physics-based extrapolation algorithms (El Hadef et al., (2012)) implemented in classical zero dimension engine models (usually implemented using commercial software or in any programming language) lead to accurate results, without increasing the calibration effort. The results for two different models are presented in this paper: first, a reference simulator implemented using the commercial software LMS AMESim, then, a Matlab code designed to be embedded in a control law.

\section{ENGINE TECHNICAL DEFINITION}

The work is based on a multi-points injection $1.2 \mathrm{~L}$ turbocharged spark-ignited engine (see figure 1). Such a light technical definition increases the turbocharger importance.
As a consequence, it makes possible to estimate the benefit induced by the new data-maps in control-oriented models.

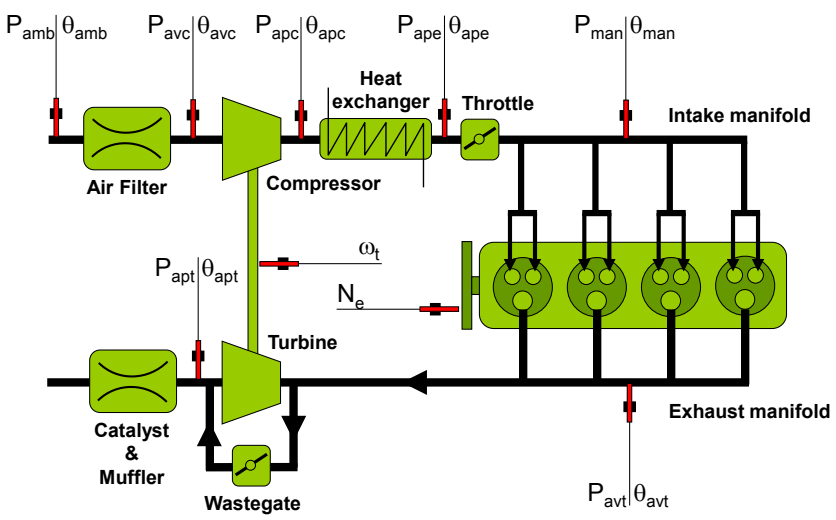

Fig. 1. Engine and sensors configuration used for the study. The engine is a turbocharged four-cylinder spark-ignited engine. Engine air mass flow rate and actuators actual positions are also recorded (except for the wastegate).

Injection and throttle control and response have been recorded. The wastegate actual position could not be measured on the engine available for the study.

Pressure and temperature before and after each air path component have been acquired. The engine rotational speed and torque as well as the turbocharger rotational speed have also been measured.

\section{REFERENCE SIMULATOR}

A OD mean value model has been developed to be used as a virtual test bench for the control development stage. Most of the components are taken from the IFP engine library. The 
others are part of the mechanical and signal AMESim library, included in the standard package.

This model has been validated in steady state conditions as well as for transients. As such, it can be used to validate the control law using, for example, hardware in the loop testing.

\subsection{Mean value engine model}

A mean value engine model component provides the air mass flow rate, the engine torque, the friction torque and the energy at the exhaust. All these outputs are estimated from data-maps which can be determined from the physical quantities available for the study (see figure 1).

This sub-model can be pre-validated by setting inlet and exhaust manifold pressures, the intake manifold temperature, the air-fuel ratio and the engine speed. In those conditions, the sub-model must already provide the right flow rate, torque and exhaust manifold temperature.

\subsection{Air path calibration}

The air path of the model contains component sub-models for the air filter, the catalyst and the muffler. They are all based on a flow restriction model. The effective cross section parameter is calibrated to match the test bench data points.

For the throttle and the wastegate, a flow restriction model is also used. In the first case, the effective area is known for every position of the actuator. For the wastegate, a PI controller determines the effective area which matches the intake manifold pressure.

The heat exchanger is modelled as the combination of a standard heat exchanger and a flow restriction. The first one is set to match the intake manifold temperature test bench data points. The second one is calibrated to match the pressure drop measured on the test bench.

The compressor and turbine models both rely on data-maps for pressure ratio, flow rate and efficiency. These data-maps are extrapolated from manufacturer's steady state data points. An innovative physical-based extrapolation strategy has been developed and is presented in section 5 (El Hadef et al., (2012)).

Compressor and turbine models are mechanically linked by a shaft which inertia is supposed to be known.

\section{CONTROL EMBEDDED MODEL}

A control embedded model must combine accuracy and stability while keeping a low calculation time. In this case, a 0D approach combined with a mean value cylinders model usually appears to be the most appropriate (Moulin et al., (2008)). The model described below is a four-state 0D model which has been validated on steady state operations as well as on transients.

\subsection{Model philosophy}

The strategy used here associates to each pipes a control volumes (see figure 2). Each of them represents a state of the model and as such, its dynamics is governed by a differential equation. Between each of them, an orifice (usually a flow restriction) controls the flow rate at the inlet (respectively at the outlet) of the control volume (see figure 2).

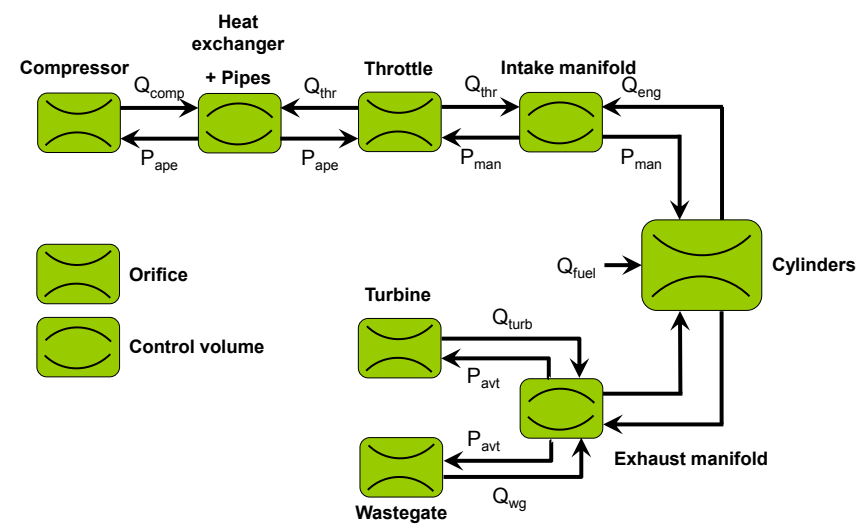

Fig. 2. Air path discretization: control volumes and restrictions.

In this model, the throttle and the wastegate are treated as flow restriction, while a data-map based model is used for the compressor and the turbine. In order to validate it, the same innovative data-maps construction as for the reference simulator is used here and detailed in section 5 .

\subsection{Reservoir model}

In each control volume $V$, Euler's mass, energy and momentum equations are applied under the assumption of static pressure. This leads to the simplified Euler's equations system. Using SI units the set of equations is given by:

$$
\begin{gathered}
\frac{\partial m}{\partial t}=Q_{m_{\text {in }}}-Q_{m_{\text {out }}} \\
\frac{\partial U}{\partial t}=Q_{m_{\text {in }}} h_{\text {in }}-Q_{m_{\text {out }}} h_{\text {out }} \\
\frac{\partial Q_{m}}{\partial t}=\frac{1}{V}\left(P_{\text {in }}-P_{\text {out }}\right)
\end{gathered}
$$

where $m$ is the mass, $Q_{m}$ the mass flow rate, $U$ the internal energy, $h$ the enthalpy and $P$ the pressure. Indices " $i n$ " and "out" respectively stand for inlet and outlet of the considered control volume.

The enthalpy flow can be approximated by:

$$
Q_{m} h=Q_{m} C_{p} \theta
$$

where $C_{p}$ is the specific heat at constant pressure and $\theta$ the temperature.

Then, from (2) and (4), the derivative of the internal energy is given by:

$$
\frac{\partial U}{\partial t}=Q_{m_{\text {in }}} C_{p_{\text {in }}} \theta_{\text {in }}-Q_{m_{\text {out }}} C_{p_{\text {out }}} \theta_{\text {out }}
$$

where $U$ is the internal energy.

It is also linked to the pressure derivative:

$$
\frac{\partial P}{\partial t}=\frac{\gamma-1}{V} \frac{\partial U}{\partial t}
$$

where $\gamma$ is the ratio of specific heats. 
It leads to the final state equation which, under the assumption of constant temperature in the reservoir (Hendricks, (2001)), governs the dynamic of the control volume:

$$
\frac{\partial P}{\partial t}=\frac{\gamma r}{V}\left(Q_{m_{\text {in }}}-Q_{m_{\text {out }}}\right) \theta
$$

where $r$ is the fluid gas constant.

The specific heat at constant pressure is defined as:

$$
C_{p}=\frac{\gamma r}{\gamma-1}
$$

As described in figure 2, the model contains three control volumes: the heat exchanger, the intake manifold and the exhaust manifold. In each of them, the pressure dynamic is computed using (7).

\subsection{Orifice model}

Inlet and outlet flow rates of control volumes are controlled by the orifices which separate them. For the throttle and the wastegate, a flow restriction model is used (Moulin et al., (2008)).

The flow is supposed to be compressible and isentropic. Under this hypothesis, the flow can be estimated using the pressure upstream and downstream the orifice (Heywood, (1988), Talon, (2004)):

$\left\{\begin{array}{l}Q_{m}=\frac{P_{u s}}{\sqrt{r T_{u s}}} A_{\text {eff }} \sqrt{\gamma}\left(\frac{2}{\gamma+1}\right)^{\frac{\gamma+1}{2(\gamma-1)}} \quad \text { if } \quad \frac{P_{d s}}{P_{u s}} \geq\left(\frac{2}{\gamma+1}\right)^{\frac{\gamma}{\gamma-1}} \\ Q_{m}=\frac{P_{u s}}{\sqrt{r T_{u s}}} A_{\text {eff }}\left(\frac{P_{d s}}{P_{u s}}\right)^{\frac{1}{\gamma}} \sqrt{\frac{2 \gamma}{\gamma-1}\left(1-\left(\frac{P_{d s}}{P_{u s}}\right)^{\frac{\gamma-1}{\gamma}}\right)} \text { otherwise }\end{array}\right.$

where $A_{\text {eff }}$ is the effective area of the orifice. The indices " $u s$ " and " $d s$ " respectively stand for upstream and downstream.

\subsection{Temperatures}

To establish (7), a constant temperature hypothesis has been done. This is the result of the fact that the dynamic of the temperature is considered to be slower than the pressure one. One can then consider:

$$
\frac{\partial \theta}{\partial t}=0
$$

As a result, the temperature in each reservoir can be computed algebraically. Many models exist in literature and depend on the considered volume. The one chosen here will be detailed on a case-by-case basis in the next sub-sections.

\subsection{Compressor model}

The compressor is considered as a flow rate source. The flow rate is read in a data-map $f_{1}$ provided by the manufacturer and extrapolated as detailed in section 5:

$$
Q_{\text {comp }}=f_{1}\left(\pi_{\text {comp }}, \omega_{t}\right)
$$

where $Q_{\text {comp }}$ is the compressor outlet mass flow rate, $\pi_{\text {comp }}$ the compression ratio and $\omega_{t}$ the turbocharger rotational speed.
The flow is distributed at a given temperature which depends on the compressor isentropic efficiency $\eta_{\text {comp }}$. It is computed algebraically using:

$$
\theta_{a p c}=\theta_{a m b}\left(\frac{\pi_{c o m p} \frac{\gamma-1}{\gamma}-1}{\eta_{c o m p}}+1\right)
$$

where $\theta_{a p c}$ is the temperature downstream the compressor, $\theta_{a m b}$ the atmospheric temperature.

The isentropic efficiency of the compressor is read in a second data-map $f_{2}$, also extrapolated from manufacturer's data:

$$
\eta_{\text {comp }}=f_{2}\left(Q_{c o m p}, \omega_{t}\right)
$$

\subsection{Turbine model}

The turbine is modelled as a flow restriction which flow rate is directly read from a data-map $f_{3}$ :

$$
Q_{\text {turb }}=f_{3}\left(\pi_{\text {turb }}, \omega_{t}\right)
$$

where $Q_{t u r b}$ is the turbine flow rate and $\pi_{t u r b}$ the expansion ratio.

The temperature of the flow at the outlet of the turbine can be obtained from the turbine isentropic efficiency:

$$
\theta_{\text {turb }}=\theta_{\text {avt }}\left[1-\eta_{\text {turb }}\left(1-\left(\frac{1}{\pi_{\text {turb }}}\right)^{\frac{\gamma-1}{\gamma}}\right)\right]
$$

where $\theta_{\text {turb }}$ is the turbine outlet temperature, $\theta_{\text {avt }}$ the exhaust manifold temperature and $\eta_{\text {turb }}$ the turbine isentropic efficiency.

As for the compressor, the turbine isentropic efficiency is read in a data-map $f_{4}$ :

$$
\eta_{t u r b}=f_{4}\left(\pi_{t u r b}, \omega_{t}\right)
$$

\subsection{Mechanical turbocharger model}

The particularity of the compressor and the turbine, as flow sources, is that they are mechanically linked. Neglecting frictions, the dynamical behaviour of the turbocharger is given by a fourth state equation which complete the model (Chauvin et al., (2011), Moulin et al., (2008)):

$$
\dot{\omega}_{t}=\frac{1}{J}\left(T_{q_{t u r b}}-T_{q_{\text {comp }}}\right)
$$

where $J$ is the turbocharger inertia, $T_{q_{\text {turb }}}$ and $T_{q_{\text {comp }}}$ respectively represent the turbine and compressor torques.

Compressor and turbines torques are computed using the model described above. In both cases, they depend on the mass flow rate, the inlet and outlet temperature and the turbocharger rotational speed:

$$
\begin{aligned}
& T_{q_{\text {comp }}}=\frac{Q_{c o m p} \times C_{p} \times\left(\theta_{a p c}-\theta_{a m b}\right)}{\omega_{t}} \\
& T_{q_{\text {turb }}}=\frac{Q_{\text {turb }} \times C_{p} \times\left(\theta_{a v t}-\theta_{t u r b}\right)}{\omega_{t}}
\end{aligned}
$$




\subsection{Mass flow rate and volumetric efficiency}

The flow rate $Q_{\text {eng }}$ is defined as a function of the intake manifold pressure and temperature as well as the engine speed (Heywood, (1988), Moulin et al., (2008)):

$$
Q_{\text {eng }}=\frac{P_{\operatorname{man}} V_{c y l}}{r \theta_{\operatorname{man}}} \frac{N_{e}}{120} \times \eta_{v o l}
$$

where $Q_{e n g}$ is the engine flow rate, $P_{\text {man }}$ and $\theta_{\text {man }}$ the manifold pressure and temperature, $V_{c y l}$ the engine displacement, $N_{e}$ the engine rotational speed and $\eta_{v o l}$ the volumetric efficiency.

The strategy consists to first calculate the theoretical mass flow rate at intake manifold conditions, under the hypothesis of a perfect gas. This quantity is then multiplied by the volumetric efficiency $\eta_{v o l}$ which represents the ability of the engine to aspire this quantity of air from the manifold. This ability directly depends from the geometry of the engine and the operating points:

$$
\eta_{v o l}=f_{5}\left(N_{e}, \frac{P_{\text {man }}}{T_{\text {man }}}\right)
$$

where $f_{5}$ is a second order polynomial calibrated on the steady state test bench measurements (average relative error is $1.7 \%$ with a standard deviation of $1.4 \%$ while maximum relative error is $8.9 \%$ ).

\subsection{Exhaust mass flow rate}

At the outlet of the cylinders, the flow rate is the sum of the inlet mass flow rate described above and the fuel mass flow rate. The last one, if not known, can be computed using the air-fuel ratio AFR:

$$
Q_{\text {fuel }}=Q_{\text {eng }} \times \frac{A F R}{14.7}
$$

where $Q_{f u e l}$ is the fuel mass flow rate and $A F R$ the air-fuel ratio.

\subsection{Exhaust enthalpy flow rate and exhaust temperature}

As underlined in Eriksson, (2007), when considering turbocharged engines, the exhaust enthalpy flow rate is essential. In fact, it represents the potential power that can be recovered by the turbine and as such, influences the intake air charge.

The exhaust manifold temperature is computed using the inlet gas conditions (mass flow rate and temperature) and the fuel mass flow rate:

$$
\theta_{\text {avt }}=\theta_{\text {man }}+k_{\text {ech }} \frac{Q_{\text {fuel }} \times L H V}{C_{p}\left(Q_{\text {fuel }}+Q_{\text {eng }}\right)}
$$

where $L H V$ is the lower heating value and $k_{e c h}$ represents the amount of energy which is transferred to the exhaust pipes flow. A polynomial model of second order is used to compute this quantity for every operating point:

$$
k_{\text {ech }}=f_{6}\left(N_{e}, Q_{\text {fuel }}, Q_{\text {eng }}\right)
$$

where $f_{6}$ is a second order polynomial which coefficients are calibrated from steady state test bench data points (average relative error is $1.8 \%$ with a standard deviation of $1.4 \%$ while maximum relative error is $6.3 \%$ ).

\subsection{Summary}

The model is described by four differential equations. Three of them concern the pressure dynamic in the control volumes and are of the form of (7). The last one describes the turbocharger dynamic (see (17)).

For computation time consideration, the use of a discrete form is highly recommended to compute the variables at step $k+1$ from values at step $k$ :

$$
\left\{\begin{array}{l}
P_{a p e}^{k+1}=P_{a p e}^{k}+\frac{\gamma r}{V_{a p e}}\left(Q_{c o m p} \theta_{a p c}-Q_{t h r} \theta_{\text {ape }}\right) \Delta t \\
P_{\text {man }}^{k+1}=P_{\text {man }}^{k}+\frac{\gamma r}{V_{\text {man }}} \theta_{\text {man }}\left(Q_{\text {thr }}-Q_{\text {eng }}\right) \Delta t \\
\mathrm{P}_{\mathrm{avt}}^{k+1}=P_{a v t}^{k}+\frac{\gamma r}{V_{\text {avt }}} \theta_{\text {avt }}\left(Q_{\text {eng }}+Q_{f u e l}-Q_{t u r b}-Q_{w g}\right) \Delta t \\
\omega_{\mathrm{t}}^{k+1}=\omega_{t}^{k}+\frac{1}{J}\left(T_{q_{\text {turb }}}-T_{q_{\text {comp }}}\right) \Delta t
\end{array}\right.
$$

where $V_{a p e}, V_{\operatorname{man}}$ and $V_{\text {avt }}$ respectively represent the volume between the compressor and the throttle, the volume of the intake manifold and the exhaust manifold volume (see figure 2). $Q_{t h r}$ and $Q_{w g}$ stand for the throttle and wastegate flows, both obtained with (9). $\Delta t$ is the sampling time and equal to 1 ms.

\section{TURBOCHARGER DATA-MAPS EXTRAPOLATION}

Most turbocharger models, which can be found in literature, are based on data-maps. However, the data-maps provided by turbocharger manufacturers usually only contain few points at high iso-speeds (data points are usually only provided for iso-speeds greater than $40 \%$ of the maximum turbocharger rotational speed). That's why, in order to simulate realistic driving cycles, the information at lower rotational speeds must be extrapolated.

In this context, a new physical-based strategy of extrapolation has been developed in order to tackle the different problems induced by current methods (Jensen et al., (1991), Martin et al., (2009b), Moraal et al., (1999)). These algorithms are fully detailed and proven in El Hadef et al., (2012).

\subsection{Compressor pressure ratio}

For the compressor mass flow rate (see figure 3), an analysis of the general turbo machinery equations (see El Hadef et al., (2012)) has led to a new physics-based algorithm. It relies on the dimensionless head parameters $\Psi$ and flow rate $\Phi$ (Martin et al., (2009a)):

$$
\Psi=\frac{A\left(\omega_{t}\right)+B\left(\omega_{t}\right) \Phi}{C\left(\omega_{t}\right)-\Phi}
$$

where the head parameter $\Psi$ and the dimensionless flow rate $\Phi$ are respectively a normalisation of the pressure ratio $\pi_{\text {comp }}$ and the mass flow rate $Q_{\text {comp }}$ and $A, B$ and $C$ are fitted using gradient optimization algorithm on manufacturer's data points.

Using monotone piecewise cubic interpolation has demonstrated very accurate results in this case (Draper et al., (1998), Fritsch et al., (1980)). 


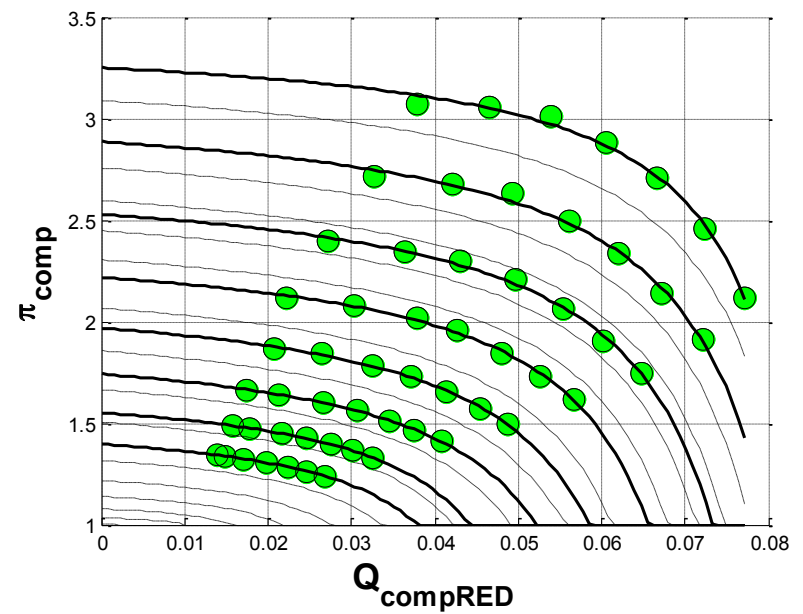

Fig. 3. Compression ratio $\pi_{\text {comp }}$ versus reduced mass flow rate $\mathrm{Q}_{\text {compRED. For each supplier's iso-speed, the pressure ratio }}$ is plotted (thick lines) and compared to the manufacturer's points (green dots). New iso-speeds, interpolated and extrapolated, are also presented (thin lines).

Another advantage of the model presented here is that (26) can directly be inverted to compute the exact inverted data map which is required in (11). In fact, one can easily write:

$$
\Phi=\frac{C\left(\omega_{t}\right) \Psi-A\left(\omega_{t}\right)}{B\left(\omega_{t}\right)+\Psi}
$$

\subsection{Compressor isentropic efficiency}

The isentropic efficiency of the compressor $\eta_{\text {comp }}$ (see figure 4) is given by the ratio of the isentropic specific enthalpy exchange $\Delta h_{i s}$ and the specific enthalpy exchange $\Delta h$ :

$$
\eta_{\text {comp }}=\frac{\Delta h_{i s}}{\Delta h}
$$

When the head parameter has been extrapolated with (26), the isentropic specific enthalpy exchange can be directly deduced through the entire operating range:

$$
\Delta h_{i s}=\frac{1}{2} \Psi U_{c}^{2}
$$

where $U_{c}$ is the blade tip speed :

$$
U_{c}=\frac{\pi}{60} D_{c} \omega_{t}
$$

where $D_{c}$ is the wheel diameter.

One can notice that the improvements achieved on the extrapolation of the expansion ratio have a direct influence here.

For the specific enthalpy exchange, Martin has proven that it is described by a linear equation (Martin et al., (2009a), Martin et al., (2009b)), particularly adapted to be fitted:

$$
\Delta h=b\left(\omega_{t}\right)-a\left(\omega_{t}\right) Q_{\text {compRED }}
$$

where, $a$ and $b$ are second order polynomials fitted using gradient optimization algorithm on the manufacturer's data points and $Q_{\text {compRED }}$ is the reduced compressor flow rate (Eriksson, (2007), Eriksson et al., (2002)).

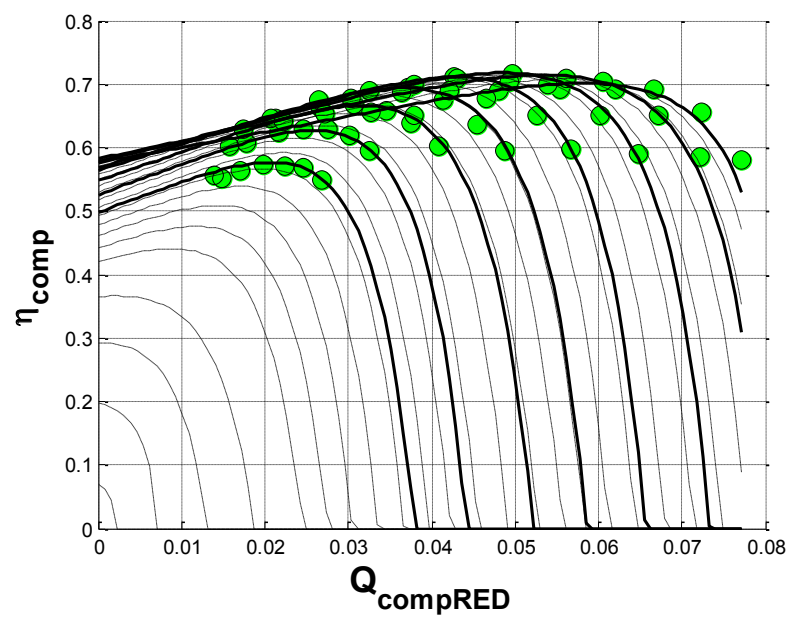

Fig. 4. Isentropic efficiency $\eta_{\text {comp }}$ versus reduced mass flow rate $\mathrm{Q}_{\text {compRED. }}$ The extrapolated compressor efficiency (thick lines) well suits to the manufacturer's data points (green dots) through the entire flow rate range. New iso-speeds, interpolated and extrapolated, are also presented (thin lines).

\subsection{Turbine pressure ratio}

In literature, the turbine is usually modelled as a flow restriction. Its flow rate (see figure 5) is given by the standard equations of compressible gas flow through an orifice (Moulin et al., (2008)):

$$
Q_{\text {turbRED }}=A \times V_{n s}
$$

where $Q_{\text {turbRED }}$ is the reduced turbine mass flow rate (Eriksson, (2007), Eriksson et al., (2002)), $A$ the equivalent section and $V_{n s}$ the reduced flow speed which depends of the flow state (subsonic or supersonic, see (9)).

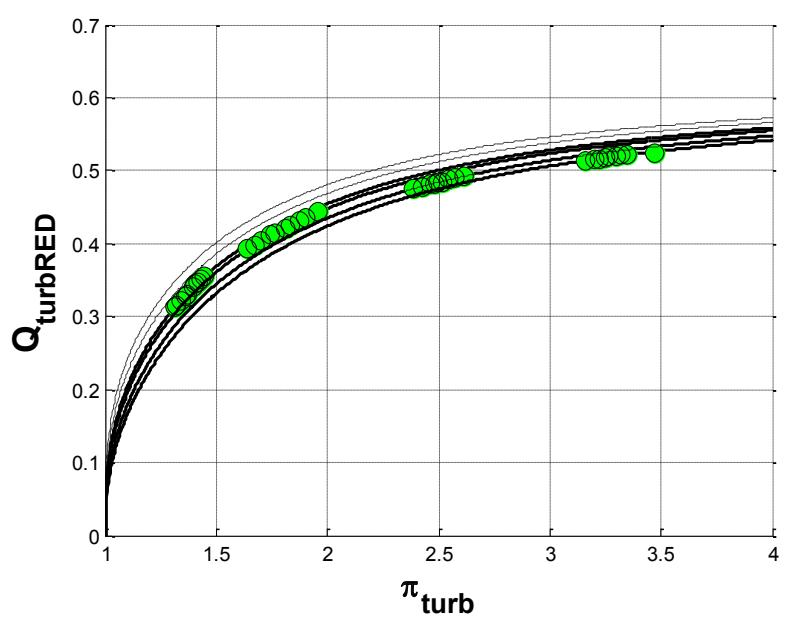

Fig. 5. Extrapolated reduced flow rate $Q_{\text {turbRED }}$ versus pressure ratio $\pi_{t u r b}$. For each manufacturer's iso-speed, the turbine flow rate extrapolated through the whole pressure ratio operating range is presented (thick line) as well as the reference points that have been used to fit the model (green dots). New iso-speeds, interpolated and extrapolated, are also presented (thin lines).

The performance of such a model essentially relies on the definition that is given to the equivalent section $S$. Definitions used in literature (Jensen et al., (1991), Martin et 
al., (2009b), Moraal et al., (1999)) usually show good performance locally (i.e. around the manufacturer's data points). However, they also suggest that the flow rate tend to infinite at high pressure ratio. This is not what is observed experimentally. In fact, from experimental observations, one can define three hypotheses for the evolution of the equivalent section with respect to the reduced mass flow rate defined in (32):

$\mathrm{H}_{1}: S$ is strictly monotonic with $\pi_{\text {turb }}$

$\mathrm{H}_{2}: \lim _{P_{i t} \rightarrow 1} S=0$

$\mathrm{H}_{3}: \lim _{P_{i t} \rightarrow+\infty} S=$ constant

According to these hypotheses, a completely new definition of $S$ has been proposed:

$$
S=k_{1} \times\left(1-e^{\left(1-\frac{1}{\pi_{\text {turb }}}\right)^{k_{2}\left(\omega_{t}\right)}}\right)
$$

where $k_{1}$ is a constant and $k_{2}$ a second order polynomial. Both are fitted using gradient optimization algorithm on the data provided by the manufacturer.

\subsection{Turbine isentropic efficiency}

The isentropic efficiency (see figure 6) is calculated in the same manner as for the compressor:

$$
\eta_{t u r b}=\frac{\Delta h}{\Delta h_{i s}}
$$

Under the hypothesis of constant fluid density (Vitek et al., (2006)), the specific enthalpy exchange is calculated using a linear equation (Martin et al., (2009a), Martin et al., (2009b)):

$$
\Delta h=c\left(\omega_{t}\right) Q_{t u r b R E D}+d\left(\omega_{t}\right)
$$

where $c$ and $d$ are second order polynomials calibrated from manufacturer's data points using regression analysis.

The isentropic specific enthalpy exchange only depends on the pressure ratio. It is computed with:

$$
\Delta h_{i s}=\left(1-\left(\frac{1}{\pi_{t u r b}}\right)^{\frac{\gamma-1}{\gamma}}\right) C_{p} T_{a v t}
$$

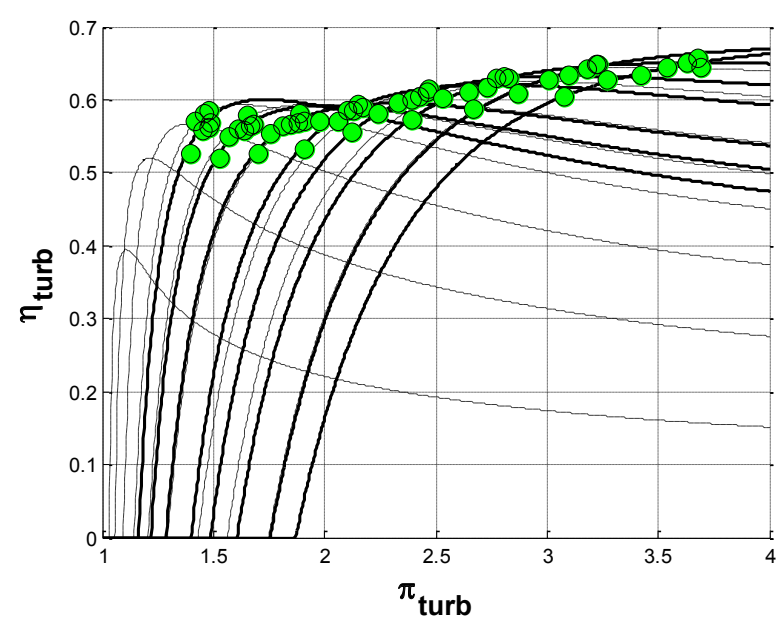

Fig. 6. Extrapolated isentropic efficiency $\eta_{\text {turb }}$. The turbine isentropic efficiency is extrapolated through the entire expansion ratio range $\pi_{t u r b}$ (thick lines) and compared to the reference values provided in the initial data-map (green dots). For these iso-speeds the model well fits to the supplier's points. New iso-speeds, interpolated and extrapolated, are also presented (thin lines).

\section{RESULTS AND DISCUSSION}

\subsection{Steady-state reference simulator performances}

As it is detailed in section 3, the building of the model is only based on steady states. About 130 points which cover the entire operating range of the engine have been used. The model performances for all these steady state points are illustrated in figures 7 to 9 .
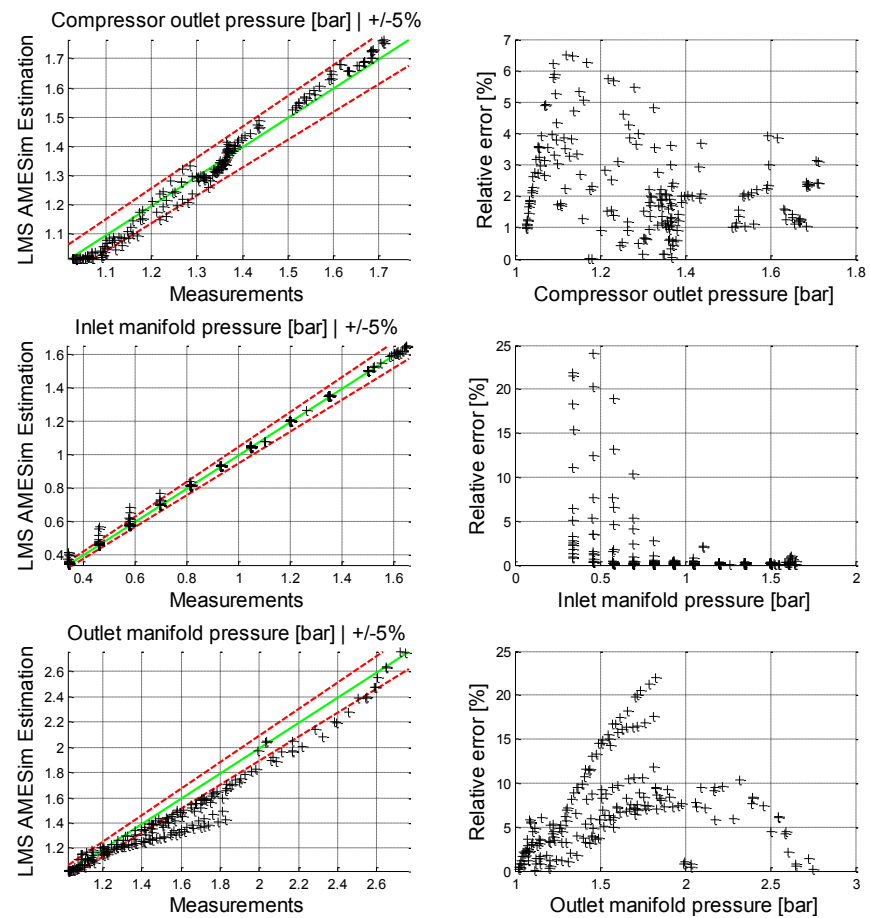

Fig. 7. Steady-states pressures validation for the reference simulator. For each physical quantity, correlation lines are plotted on the left. A perfect model would give 45 degrees tilted straight line. Dashed lines show variation zones specified in the title. Relative error versus test bench measurement is plotted on the right.
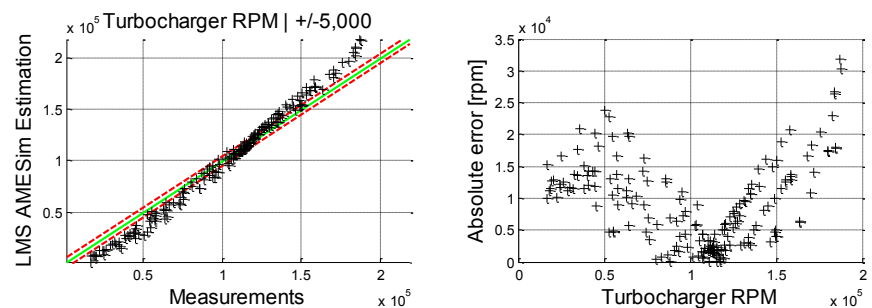

Fig. 8. Steady-states turbocharger rotational speed validation for the reference simulator. 

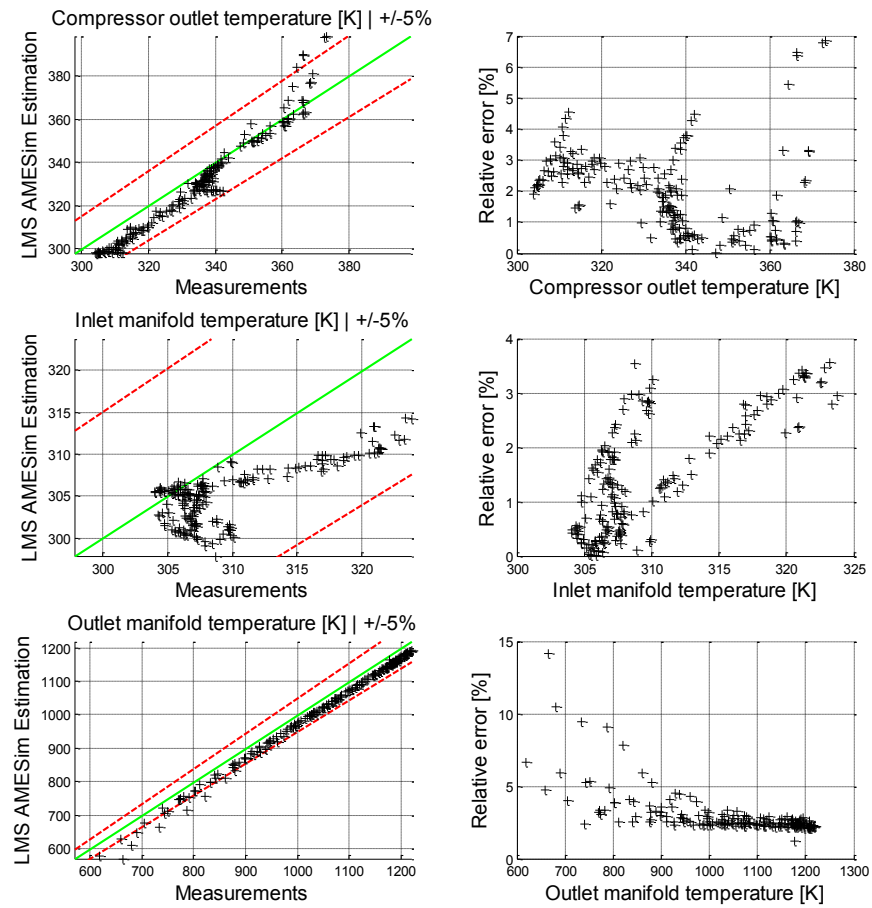

Fig. 9. Steady-states temperatures validation for the reference simulator.

\subsection{Steady-state control embedded model performances}

The control embedded model validation stage uses the same steady state operating points as for the reference simulator. All the results are presented in figures 10 to 12 .
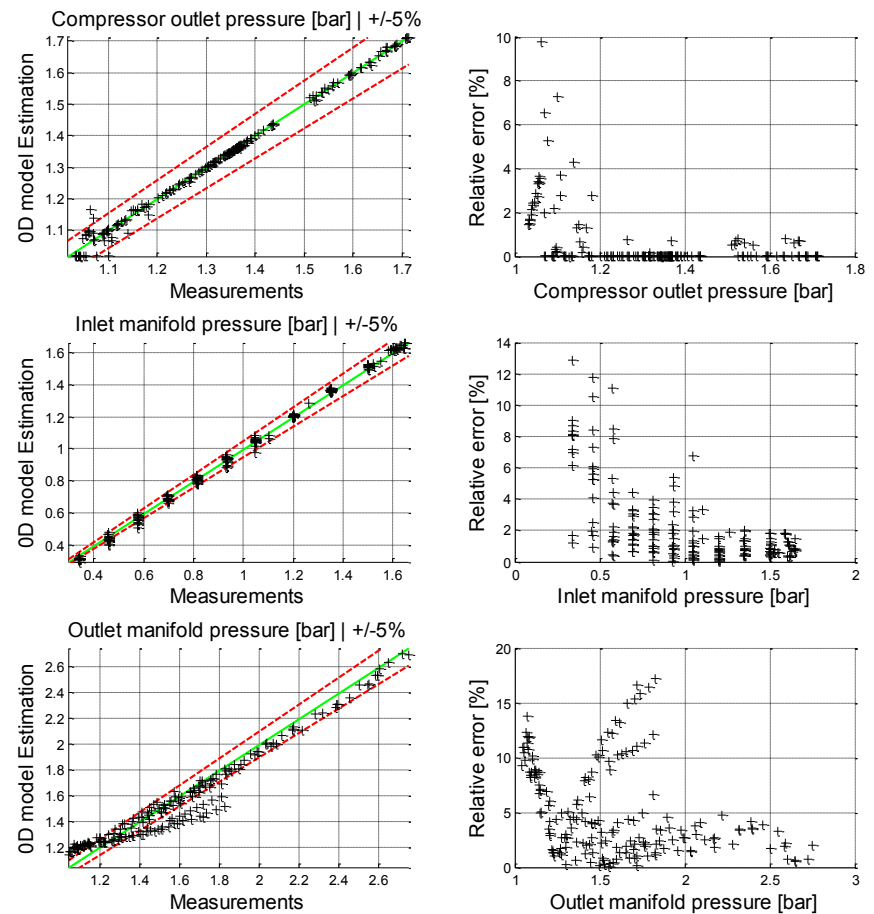

Fig. 10. Steady-states pressures validation for the control embedded model.
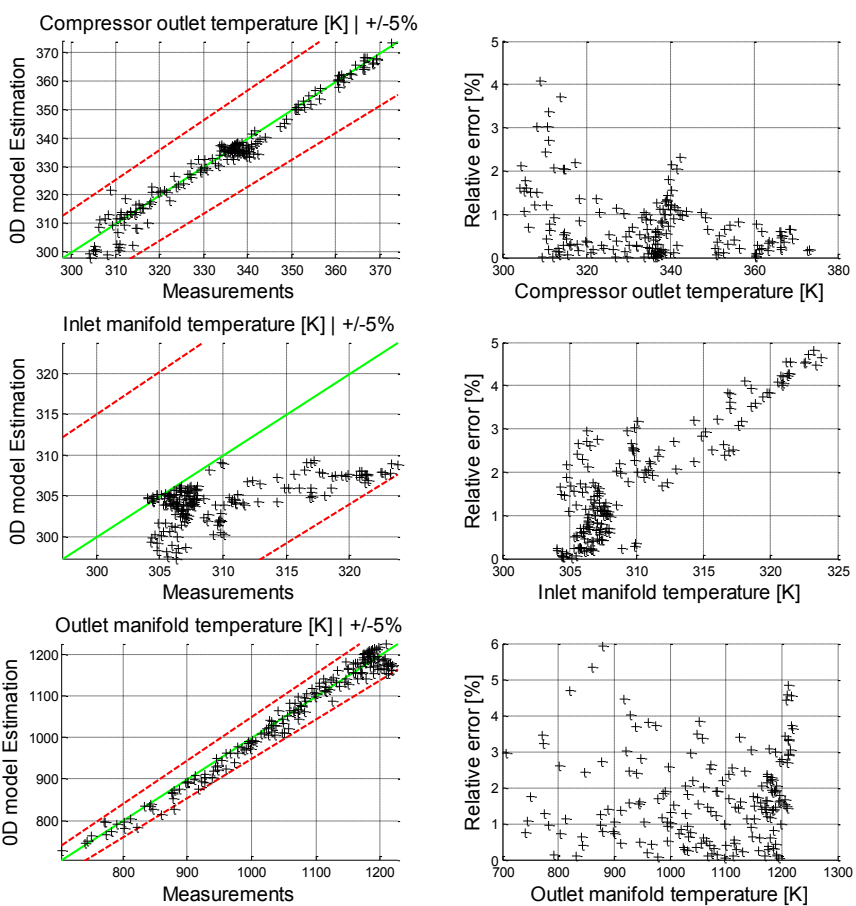

Fig. 11. Steady-states temperatures validation for the control embedded model.
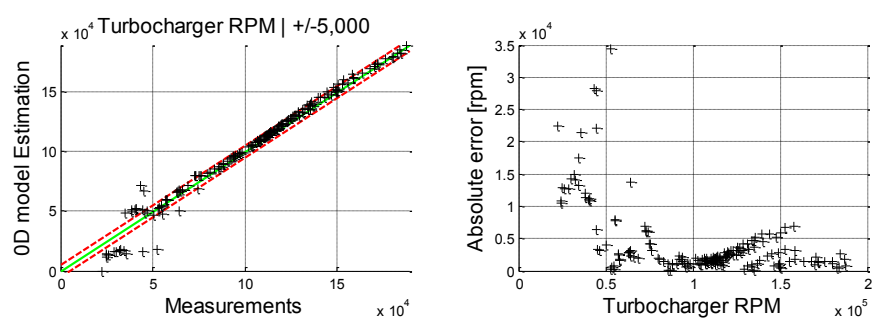

Fig. 12. Steady-states turbocharger rotational speed validation for the control embedded model.

\subsection{Discussion}

Both models basically have the same static behaviour. On figures 7 to 9 and on figures 10 to 12 , one can see that both models present a low relative error (particularly at high loads). For pressures and temperatures, the average relative error for the AMESim model is about $10 \%$. The average relative error on these values for the control embedded model is even lower. The estimation of the turbocharger speed is less accurate. The error can reach $30,000 \mathrm{rpm}$ for the reference simulator while it reaches only $25,000 \mathrm{rpm}$ for the second model at low speeds.

For control purposes, it is crucial to capture the dynamic of control variables, i.e. the pressures in the control volumes. For both models, these dynamics are well estimated (see figure 13). The relative error is less than $5 \%$ for compressor outlet and intake manifold pressures. The performance is a bit lower for the exhaust manifold pressure: the error can locally reach $20 \%$ on the transient presented here, but the dynamic is usually good. In both models, the turbocharger rotational speed dynamic is well captured (the average error is less than $9,000 \mathrm{rpm}$ ), in particular at low rotational speeds and pressure ratios, where the data are fully extrapolated. 

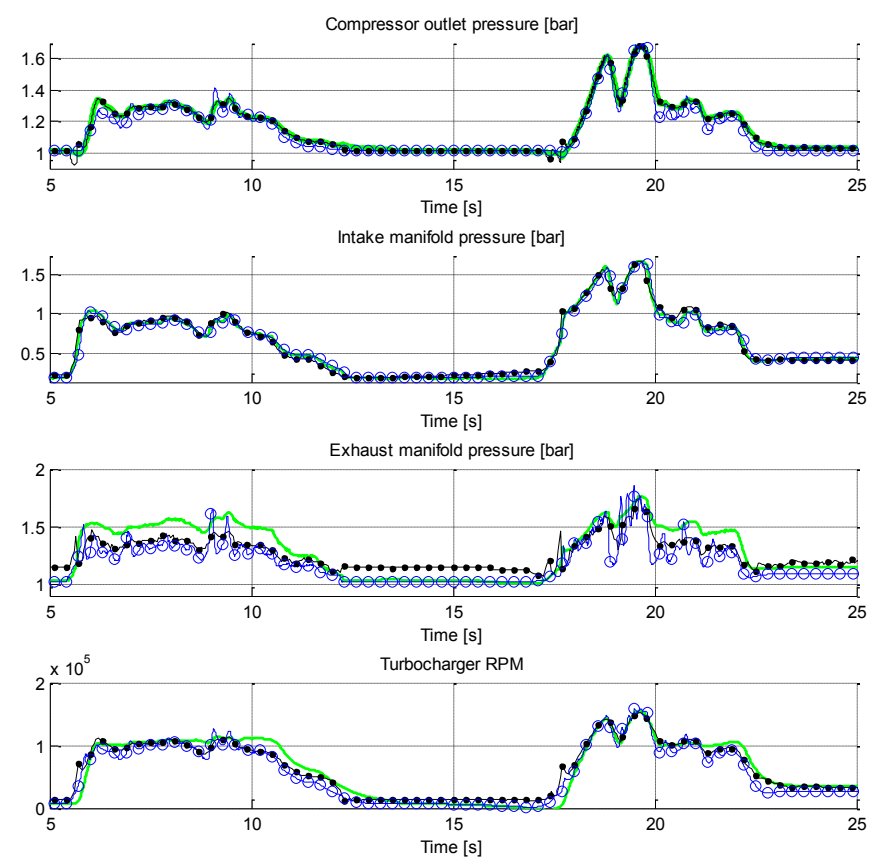

Fig. 13. Transients validation of pressures and rotational speed for the reference simulator (blue circled line) and for the control embedded model (black dotted line). Vehicle measurements are also plotted (thick light coloured line). Engine speed varies from 4,000 to $6,000 \mathrm{rpm}$ while throttle and wastegate positions vary from closed to fully opened (including sudden opening).

\subsection{Limitations}

One should notice that the difference between the measurements and the simulation results is a global error which can be addressed to three different main sources of error: the pulse effects, the thermal effects and the extrapolation algorithms. The first two are not explicitly taken into account in the model. Moreover, the part that each phenomenon has on the error cannot be evaluated with the data presented here. That is why a comparative study between a $0 \mathrm{D}$ model based on a classical extrapolation method or based on the new one is irrelevant.

The goal of this study was to show that any classical controloriented model, identified using exclusively steady states test bench measurements and based on data maps extrapolated using the new physics-based algorithms, leads to accurate enough results in the context of an industrial application.

\section{CONCLUSION}

Extrapolated turbocharger rotational speeds zone can easily represent $50 \%$ of a classical driving cycle. This study has been motivated by the difficulty encountered with standard techniques to obtain accurate data in this operating range. Thanks to an appropriate combination of physics and mathematical fitting tools, it has been shown that the new extrapolation strategy leads to accurate control-oriented engine models. The advantage is that the new algorithms are more robust than standard methods while keeping the zero dimensional approach and a low CPU load requirement.

\section{REFERENCES}

Chauvin, J., Grondin, O., and Moulin, P. (2011). Control Oriented Model of a Variable Geometry Turbocharger in an Engine with Two EGR loops. Oil \& Gas Science and Technology - Rev. IFP Energies nouvelles, 66 (4), 563-571.

Dauron, A. (2007). Model-Based Powertrain control: Many Uses, No Abuse. Oil \& Gas Science and Technology - Rev. IFP Energies nouvelles, 62 (4), 427-435.

Draper, N. R., and Smith, H. (1998). Applied Regression Analysis. Wiley.

El Hadef, J., Colin, G., Talon, V., and Chamaillard, Y. (2012). Physical-Based Algorithms for Interpolation and Extrapolation of Turbocharger Data Maps. SAE Int.J.Engines 5(2):2012, doi:10.4271/2012-01-0434

Eriksson, L. (2007). Modeling and Control of Turbocharged SI and DI Engines. Oil \& Gas Science and Technology Rev. IFP Energies nouvelles, 62 (4), 523-538.

Eriksson, L., Nielsen, L., Brugard, J., and Bergström, J. (2002). Modeling of a Turbocharged SI Engine. Annual Reviews in Control, 26, 129-137.

Fritsch, F. N., and Carlons, R. E. (1980). Monotone Piecewise Cubic Interpolation. SIAM Journal on Numerical Analysis, 17 (7).

Gissinger, G., and Le Fort-Piat, N. (2002). Contrôle Commande de la Voiture. Hermès Lavoisier.

Guzzella, L., and Onder, C. H. (2004). Introduction to Modeling and Control of Internal Combustion Engine Systems. Springer.

Hendricks, E. (2001). Isothermal versus Adiabatic Mean Value SI Engine Models. 3rd IFAC Workshop, Advances in Automotive Control, 373-378.

Heywood, J. B. (1988). Internal Combustion Engines Fundamentals. McGraw-Hill.

Jensen, J.-P., Kristensen, A. F., Sorenson, S. C., Houbak, N., and Hendricks, E. (1991). Mean Value Modeling of a Small Turbocharged Diesel Engine. SAE Technical Paper, 910070.

Martin, G., Talon, V., Higelin, P., Charlet, A., and Caillol, C. (2009a). Implementing Turbomachinery Physics into DataMap Based Turbocharger Models. SAE Technical Paper, 2009-01-0310.

Martin, G., Talon, V., Peuchant, T., Higelin, P., and Charlet, A. (2009b). Physics Based Diesel Turbocharger Model for Control Purposes. SAE Technical Paper, 2009-24-0123.

Moraal, P., and Kolmanovsky, I. (1999). Turbocharger Modeling for Automotive Control Applications. SAE, 199901-0908.

Moulin, P., Chauvin, J., and Youssef, B. (2008). Modelling and Control of the Air System of a Turbocharged Gasoline Engine. Proc. of the IFAC World Conference 2008.

Talon, V. (2004). Modélisation 0-1D des Moteurs à Allumage Commandé. PhD Thesis, Université d'Orléans.

Vitek, O., Macek, J., and Polasek, M. (2006). New Approach to Turbocharger Optimization using 1-D Simulation Tools. SAE Technical Paper, 2006-01-0438. 